\title{
EFFECT OF OVALITY IN INLET PIGTAIL PIPE BENDS UNDER COMBINED INTERNAL PRESSURE AND IN-PLANE BENDING FOR Ni-Fe-Cr B407 MATERIAL
}

\begin{abstract}
The present paper makes an attempt to depict the effect of ovality in the inlet pigtail pipe bend of a reformer under combined internal pressure and in-plane bending. Finite element analysis (FEA) and experiments have been used. An incoloy Ni-Fe-Cr B407 alloy material was considered for study and assumed to be elastic-perfectly plastic in behavior. The design of pipe bend is based on ASME B31.3 standard and during manufacturing process, it is challenging to avoid thickening on the inner radius and thinning on the outer radius of pipe bend. This geometrical shape imperfection is known as ovality and its effect needs investigation which is considered for the study. The finite element analysis (ANSYS-workbench) results showed that ovality affects the load carrying capacity of the pipe bend and it was varying with bend factor (h). By data fitting of finite element results, an empirical formula for the limit load of inlet pigtail pipe bend with ovality has been proposed, which is validated by experiments.
\end{abstract}

Keywords: ovality, inlet pigtail pipe bend, reformer, finite element analysis, in-plane bending

\section{Introduction}

Pipe bends are common throughout all piping systems which provide additional flexibility in the piping network by absorbing thermal expansion or contraction. Pipe bends employed in a reformer are subjected to various degradation mechanisms, resulting in the failure of the pipe bend region. Failure of inlet pigtail pipe bend having catastrophic effect at extrados (Fig. 1) and this failure is identified at site. During plant operation, the piping system is exposed to thermal and mechanical loads due to internal pressure, axial tension, global bending moment, combined internal pressure and bending moment. These induced loads are transmitted to the curved region, causing high stress level when compared to a straight pipe [1]. Further, during the forming process, the outer fibre of the pipe bends thin down when compared to the inner fibre, which leads to a phenomenon known as ovality and thinning [2-3].

Ovality and thinning (shape irregularities) in pipe bends induce higher stress during operation of the plant. The shape imperfections in pipe bends and the components to which they are attached beyond a certain level will damage the piping system. Besides shape irregularities, pipe diameter, wall thickness and bend radius also effect a change in stress in bend region [4]. However, the majority of the existing solutions have been developed either empirically based on test data or analytically based on a simple equilibrium stress field and yield criterion considering without the effect of ovality in pipe bends [5-10],

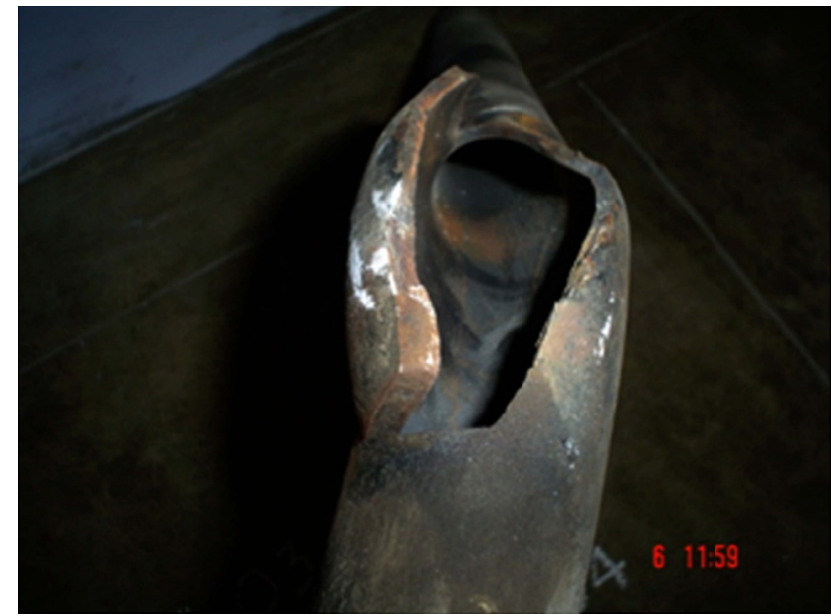

Fig. 1. Failure in inlet pigtail pipe bend

thus the resulting solutions tend to under predict the actual limit loads, which lead to failure in bend region. Effect of ovality in pipe bend should be quantified and included in limit loads solution to avoid failure.

The length of a straight pipe (five times the bend radius) was attached with pipe bend to remove the end effect caused by bending moment. It was found that the FE limit load results were much higher due to the presence of the attached straight pipe to the pipe bend than the existing analytical solution [11]. The limit load was shown decreasing with the decreasing length

\footnotetext{
** DEPARTMENT OF MECHANICAL ENGINEERING, KONGU ENGINEERING COLLEGE, ERODE, TAMILNADU, INDIA 
of the attached straight pipe and eventually to approaching the existing analytical solutions [12].

This paper quantifies the effect of ovality under combined internal pressure and in-plane bending on limit loads of the inlet pigtail pipe bends with the attached straight pipe. The detailed three dimensional finite element analyses performed using elastic perfectly plastic material and the results are validated with experiments.

\section{Finite element analysis}

\section{2a. Geometry}

Fig. 2 depicts a 90 degree pipe bend with attached with a straight pipe considered in the present work. The mean radius and thickness of the pipe bend are denoted by $r$ and $t$, respectively, and the bend radius by $R$. The pipe bend is connected with a straight a pipe of length equal to five times the bend radius [12]. The cross section of the inlet pigtail bend frequently assumes an oval shape (Fig. 3) and the percent ovality, $C_{o}$, [13] and pipe bend characteristics, $h$, [11] are defined by

$$
\begin{gathered}
C_{o}=\frac{\left(D_{\max }-D_{\min }\right)}{\left(D_{\max }-D_{\min }\right) / 2} * 100 \\
h=\frac{R t}{r^{2}}=\frac{(R / r)}{(r / t)}
\end{gathered}
$$

The above non dimensional variables were varied systematically to measure the effect of ovality in bend geometry on limit loads. Values of $r / t$ were ranged from 5 to 20, with those of bend factor h from 0.1 to 0.5 . The percent ovality $C_{o}$ varied between 0 and 20. The bend geometry is modeled with ovality (Fig. 3) for which the maximum outer diameter is $D_{\max }=D+2[\mathrm{X}]$ and minimum outer diameter $D_{\min }=\mathrm{D}-2[\mathrm{X}]$.

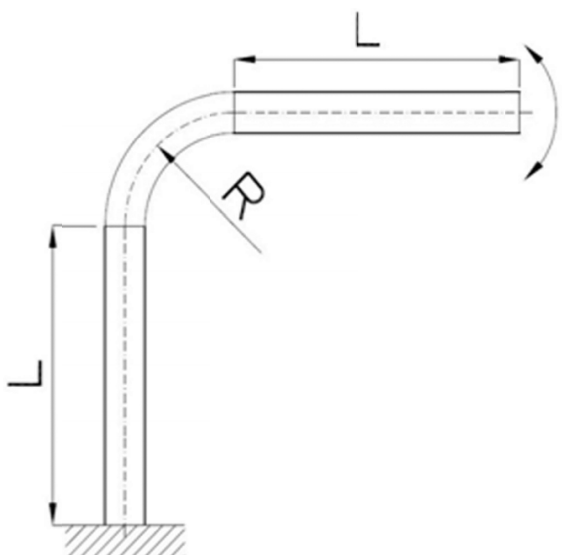

Fig. 2. Pipe bend with attached straight pipe

\section{2b. Material}

The present work includes the stress and deformation analysis of inlet pigtail pipe bend made from an incoloy $\mathrm{Ni}$ -

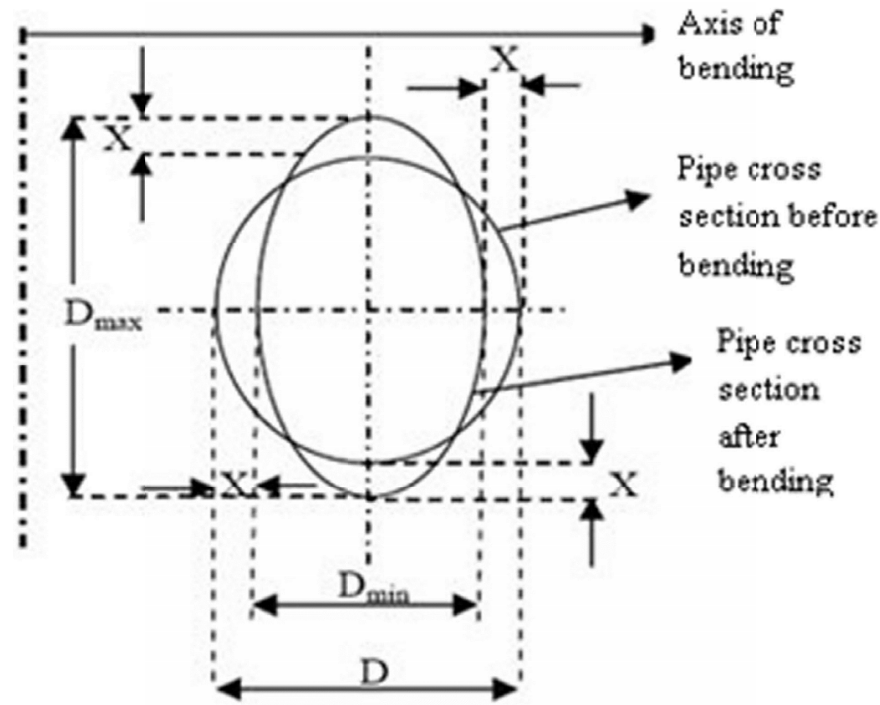

Fig. 3. Cross section of pipe bend with ovality

Fe-Cr B407 alloy material. The relevant material properties are $E=196 \mathrm{KN} / \mathrm{mm}^{2}, \gamma=0.3$, and $\sigma_{o}=172 \mathrm{KN} / \mathrm{mm}^{2}$, where $E, \gamma$ and $\sigma_{o}$ are Young's modulus, Poisson's ratio and limiting yield stress respectively.

\section{2c. FE mesh and analysis}

The geometry of inlet pigtail pipe bend was modeled in CATIA and imported in to ANSYS- Work bench as an IGES file. The workbench environment is an intuitive up-front finite element analysis tool which is used in conjunction with CAD systems and or design modeler. To establish a suitable mesh density for the FE model, a convergence study was performed and pipe bend was described by 28,139 nodes and 13,190 elements along the Pipe. The domain was applied with the boundary constraints and solved for the solution. Internal pressure of $10 \mathrm{MPa}$ was applied as a distributed load to the inner surface of the model. In addition, an axial tension equivalent to the internal pressure was applied at the end of the pipe to simulate closed end.

The von-mises stresses induced at extrados, midsection and intrados of the inlet pigtail pipe bend are significant for combined internal pressure and in-plane bending moment. For the uniform cross section, it was identified that the pipe bend gets more stabilized and stress distribution is uniform in bend geometry (Fig. 4a). When the pressure and moment is applied in the pipe bend, overall flexibility starts increasing gradually due to cross section distortion. (Fig. 4b-d) indicates the von-mises stress distribution over the pipe bend for the percent ovality 4, 8 and 12 respectively. It has been noted that induced stresses are higher compared with the pipe bend having no ovality and it is increased in the extrados region for higher percent ovality. The stress concentration in extrados region is much higher than intrados for the pipe bend with percent ovality of 16 and 20 (Fig. 4e-f). It can be noted that sudden plasticization at bend region is identified at the extrados of pipe bend. 


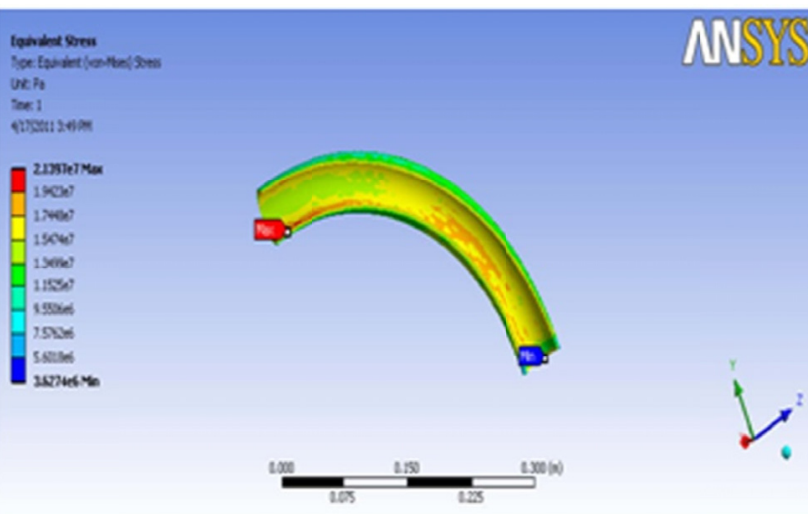

a) $0 \%$ Ovality

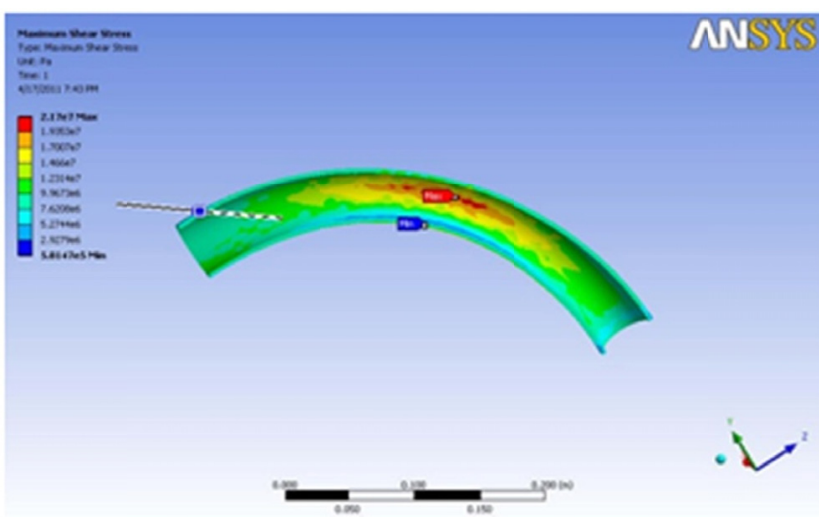

c) $8 \%$ Ovality
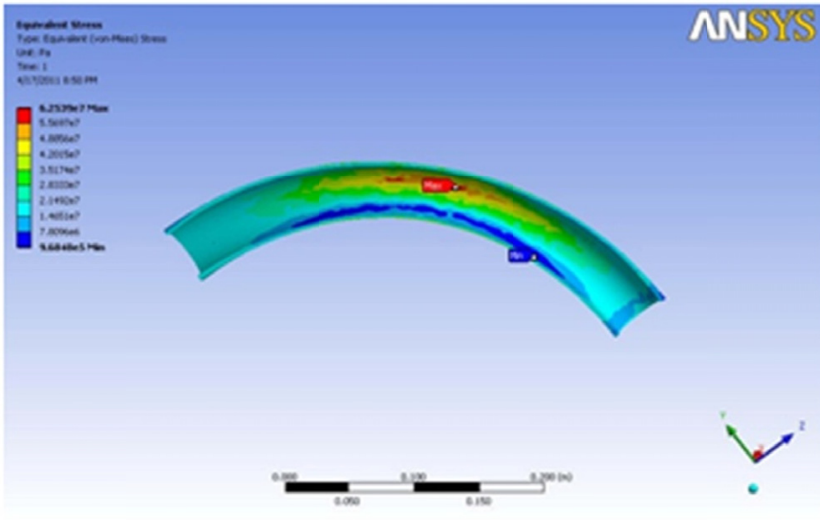

e) $16 \%$ Ovality

Fig. 4. Von-mises stresses in pipe bend geometry

\section{2d. Limit load}

Various researchers have studied the problem of limit load of pipe bend under internal pressure and in plane bending and suggested closed form expressions of the limit load. It is now well known that combined internal pressure and in plane moment have significant effect on the limit load of pipe bend [12]. The limit moment equation based on the small strain assumption is

$$
M=\left(4 r^{2} t \sigma_{o}\right)\left(1.04 h^{2 / 3}\right)\left[1-P \frac{r}{2 t \sigma_{o}}\right]^{1 / 3}
$$

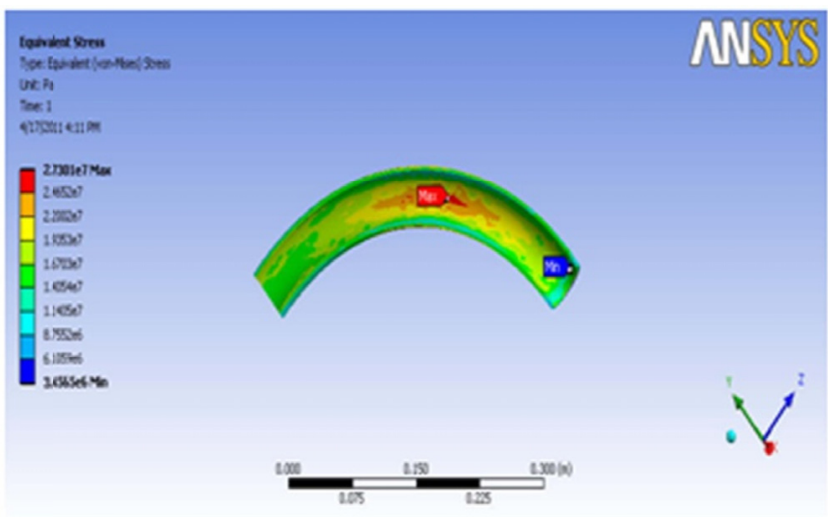

b) $4 \%$ Ovality

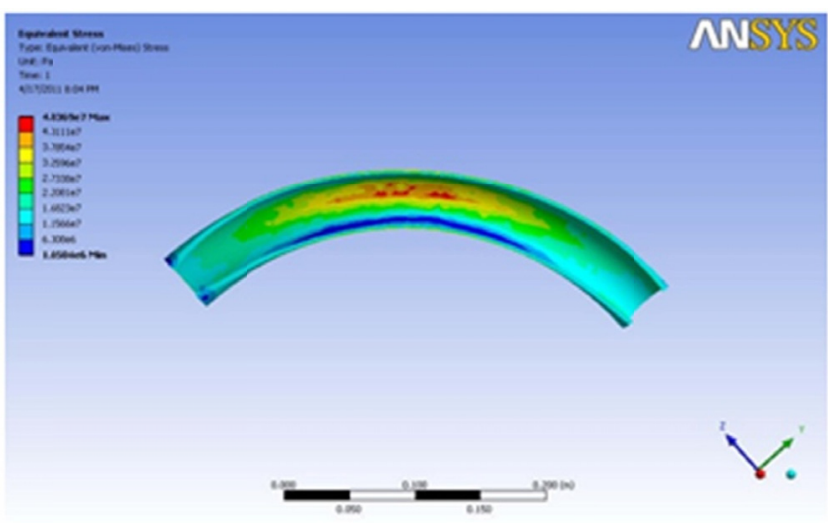

d) $12 \%$ Ovality

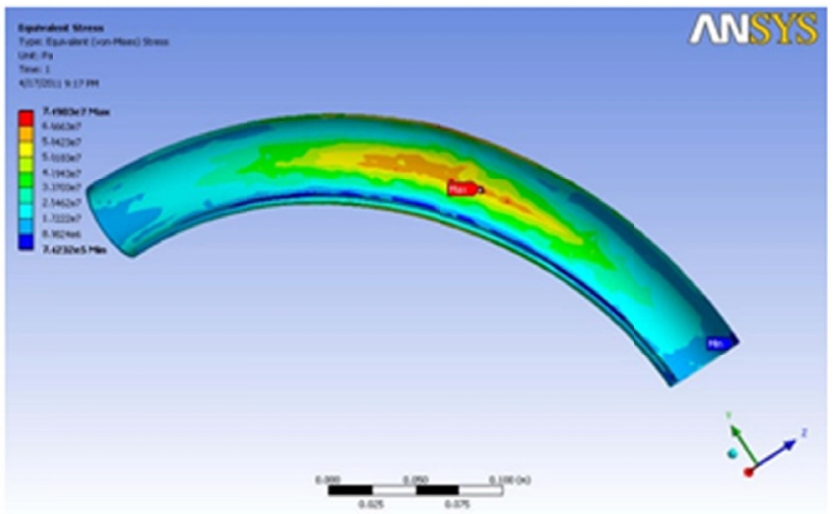

f) $20 \%$ Ovality

The first two terms in the right hand side of Eq. (3) are related to the limit load for a pipe bend under bending, and the third term reduction is due to internal pressure. However their study on pipe bends was performed relying on the assumptions that the constant wall thickness along the contour of the bends cross section.

The yield locus for the pipe bends under combined pressure and bending moment was compared with the finite element results (Fig. 5). The results are obtained for fixed $r / t$, but the value of $R / r$ is varied from 2 to 5 and the range of $h$ from 0.2 to 0.5 . For the finite element calculations, the bend with the 
attached straight pipe of $L=5 R$ was considered. The Eq. (3) is overall conservative except for the pressure dominant region. It can be noted that the pressure term accounts for only the axial stress and thus is not correct for internal pressure. The Eq. (3) could be modified to

$$
M=\left(4 r^{2} t \sigma_{o}\right)\left(1.04 h^{2 / 3}\right)\left[1-P \frac{r}{t \sigma_{o}}\right]^{1 / 3}
$$

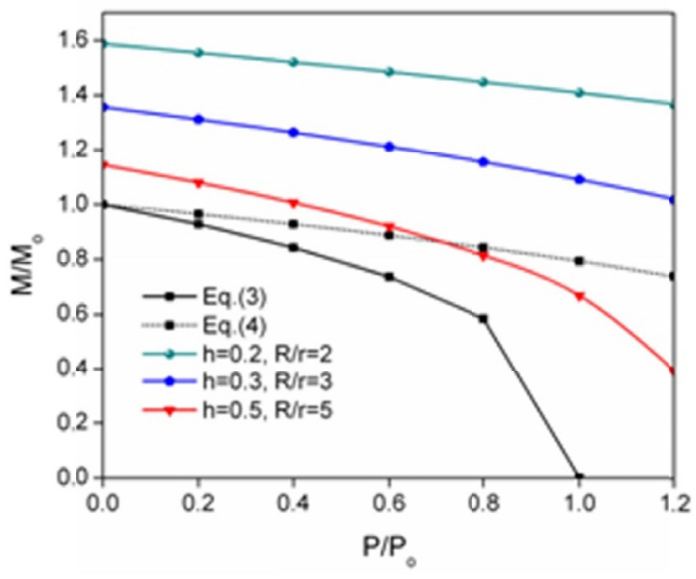

Fig. 5. Comparison of the FE results

FE limit analyses are performed based on elastic perfectly plastic material using both the small geometry change and large geometry change options. The FE results using small geometry change option suggest that the existing analytical limit solutions for pipe bends are indeed lower bounds. By data fitting of finite element results, an empirical formula for the limit load of inlet pigtail pipe bend with ovality under combined internal pressure and bending is proposed.

$$
\frac{M}{M_{o}}=1-\left(\frac{1-2 C_{o}}{1-C_{o}} \frac{1-r / R}{1-r /(2 R)} \frac{P}{P_{O}}\right)^{3}=1-\left(\frac{P}{P_{o}^{s}}\right)^{3}
$$

The value of pressure ratio $P / P_{O}$ cannot be greater than unity, the yield locus, Eq. (5) should be cut off at $P / P_{O}=1$. Based on FE results, the bending moment ratio was plotted for $R / r=2$ with bend factor of 0.2 (Fig. 6). It has been noted that the load

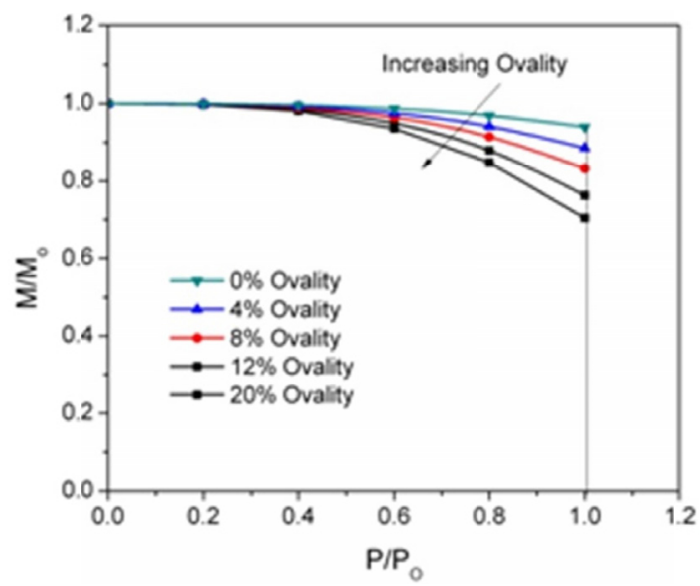

Fig. 6. Limit load variation $(h=0.2, R / r=2)$ carrying capacity of pipe bend is reduced for the higher bend factor and then it was further reduced when the percent ovality increased. The effect of bend radius is to reduce the second moment of areas of the section and therefore the moment ratio got reduced progressively (Fig. 7). The effect of ovality in limit load plotted for $R / r=5$ (Fig. 8) which shows that the limit loads are lesser for higher bend radius and percent ovality.

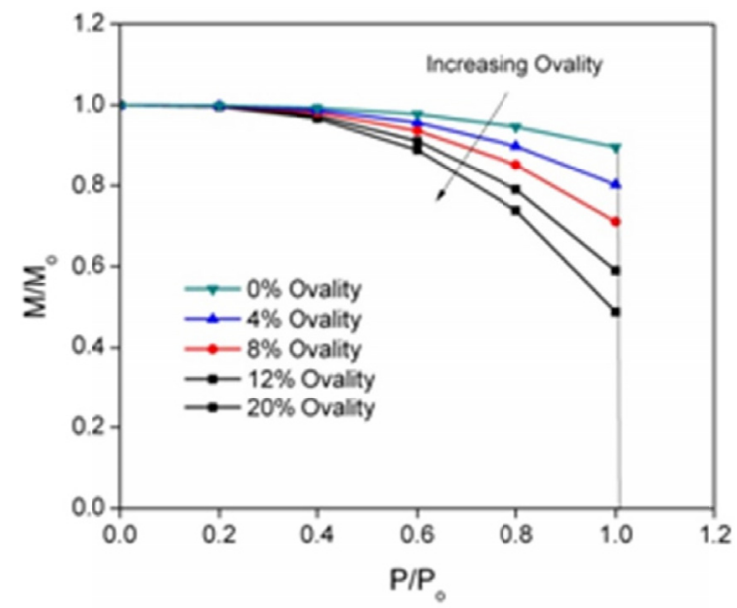

Fig. 7. Limit load variation $(h=0.3, R / r=3)$

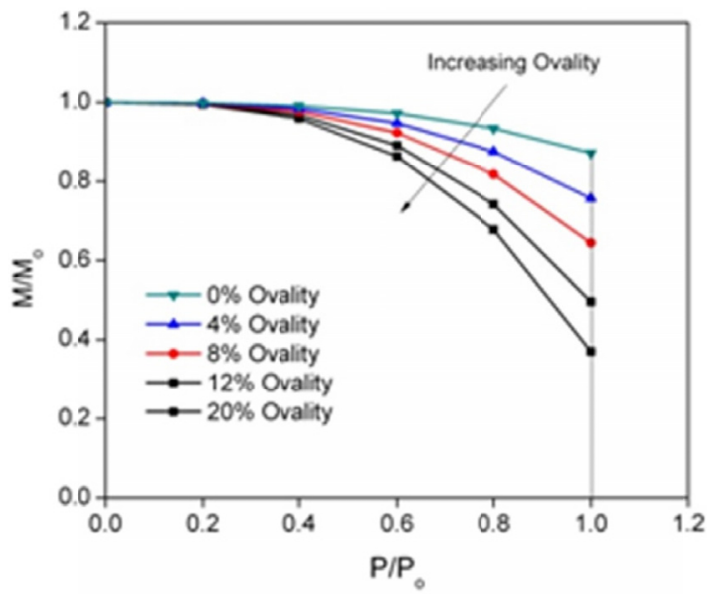

Fig. 8. Limit load variation $(h=0.5, R / r=5)$

\section{Experimental evaluation}

The diagrammatical model of the experimentation setup is shown in Fig. 9 for testing the inlet pigtail pipe bend under combined internal pressure and in-plane bending mode. Three bend specimens were arbitrarily selected from a total of nine that had been considered for a limit experimental programme.

Specimen 1: $h=0.2, R / r=2, r / t=10$

Specimen 2: $h=0.3, R / r=3, r / t=10$

Specimen 3: $h=0.5, R / r=5, r / t=10$

One end of the pipe end was fixed at the structural element and the other end was kept free for applying the in-plane moment load. A long rod of length, equal to five times the bend radius $(R)$ was attached at the free end for applying the in-plane load. Spring balance and load cell were used to measure the magnitude of the 
applying load which is placed at the free end of the rod [10]. Dial gauges were used to measure the deflection in the bend section by fixing it in the intrados, crown and extrados regions (Fig. 10).

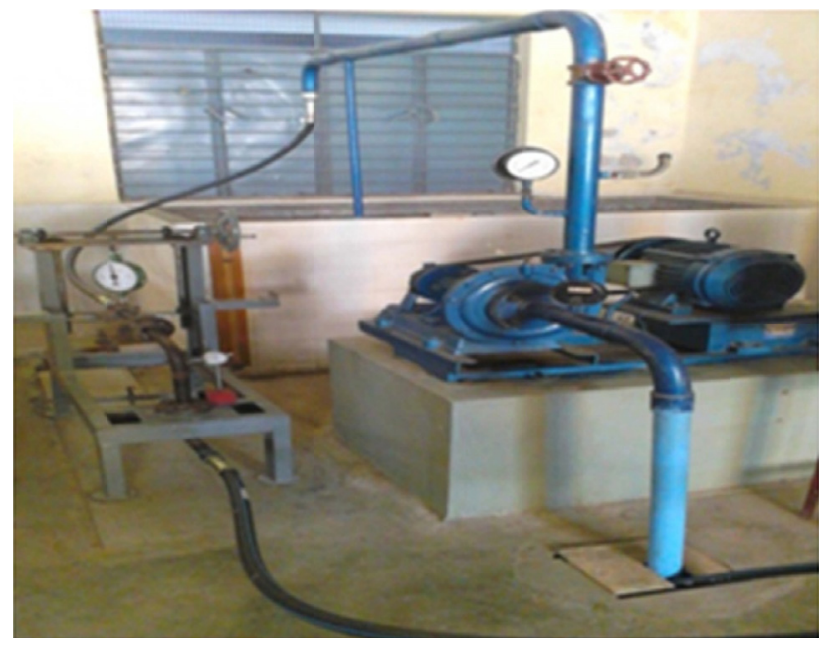

Fig. 9. Loading arrangement for pipe bends

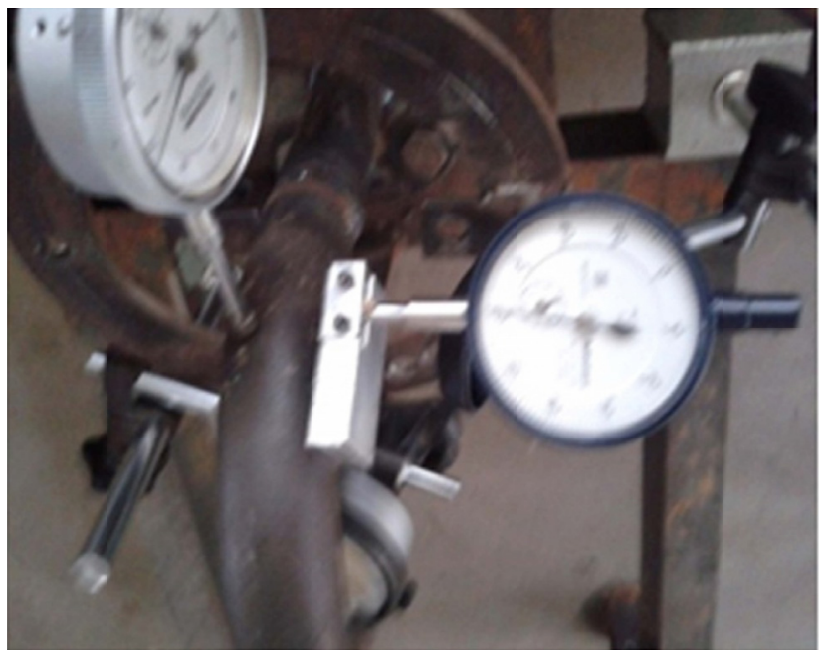

Fig. 10. Dial Gauge Arrangements

Test specimens of Ni-Fe-Cr B407 alloy material with different percent ovality are considered for the study. Internal pressure of $10 \mathrm{MPa}$ applied on bend geometry and applied load (both opening/closing mode) was incremented by $10 \mathrm{KN}$ until the bend undergoes plastic instability. Further, to keep internal pressure as a constant, the load was applied at one end of the pipe to quantify the effect of bending moment. It was ensured that the other end is firmly fixed. The applied load was converted to moment, based on the straight pipe length attached to the pipe bend. Also in the vertically downward direction, the bend section was subjected to in-plane closing mode. The displacements were measured, using a dial gauge in order to quantify the stress variation.

From these load-deflection curves, limit loads were evaluated for each pipe bend specimen by twice elastic slope (TES) method [12]. Few points were noted based on load deflection curves. Firstly, for the same bending moment (i.e. opening/ closing), a pipe bend with lesser thickness showed lower stiffness and load bearing capacity. It is quite natural that, a lesser thickness pipe bend with higher percent ovality weakens to a greater extent, which reduces its stiffness and load bearing capacity. Secondly, a pipe bend with a certain design parameter subjected to opening mode of bending moments has higher limit load, when compared to a pipe bend with the same design parameter subjected to closing moment. The same behavior has been obtained in the numerical exercises [4].

The experimental load deflection curves for in-plane closing mode loading conditions considering various design parameters were plotted (Fig. 11). When the pipe bend was subjected to closing bending moment, it showed that the load starts dropping after certain displacement. The limit loads were noted for various bend geometry based on TES method and it was found that the limit load is higher for lower bend radius. When the pipe bend was subjected to opening bending moment, it did not show the dropping nature of load-deflection curve (Fig. 12) and the limit for the specified design condition is higher than the closing mode case. Bending moment at oval section of pipe bend (opening/closing mode) at any point of time will be governed by the equation.

$$
M=\int \sigma \cdot d Z
$$

Where, $M$ is the bending moment, $\sigma$ is the local stress and $\mathrm{Z}$ is the section modulus at the cross section. As the pipe bend is loaded, the local stress $\sigma$ increased because of the strain hardening of material. However, area moment of inertia and hence the section modulus $(Z)$ decreased which is influenced by the ovalisation of pipe bend cross section. The pipe bend cross section when subjected to closing mode of bending moment ovalised in such a way that reduced the $Z$. In contrast, a pipe bend when subjected to the opening mode of bending moment has higher $\mathrm{Z}$ because of ovalisation during its deformation. In case of closing moment, $Z$ is reduced because of ovalisation. Therefore, beyond a point there is an increase in local stress $\sigma$ because strain hardening is exactly compensated by the decrease in $Z$ moment, which starts falling in case of closing moment. In case of opening moment, $Z$ increases due to ovalisation. Therefore, reduction in $Z$ is much

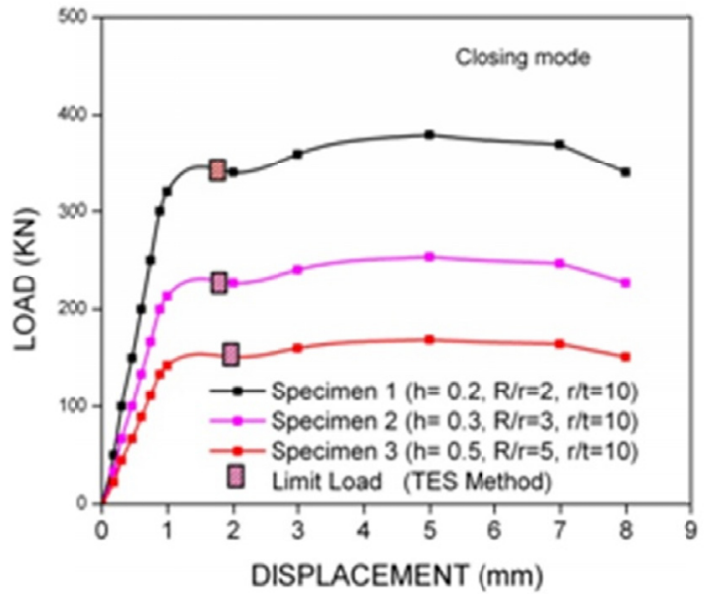

Fig. 11. Load deflection curve (closing mode) 


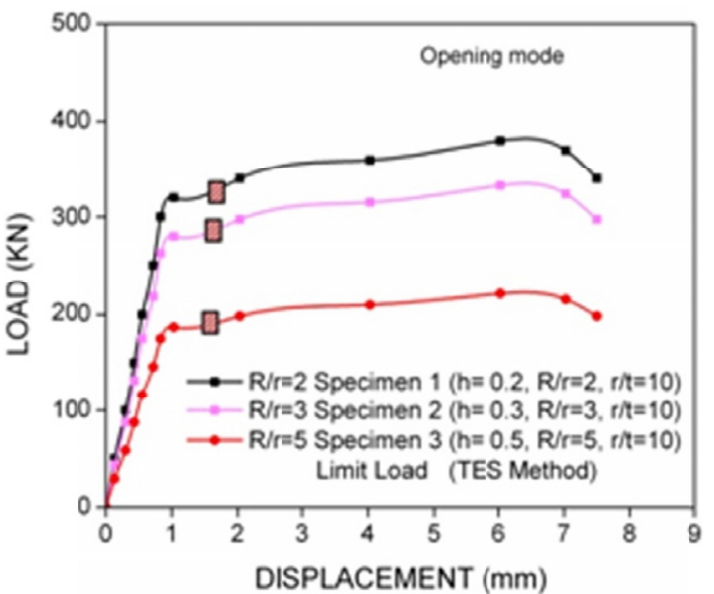

Fig. 12. Load-deflection curve (opening mode)

less or not at all present in case of opening moment and hence no drop in moment (or load) is generally observed.

Fig. 13 showed the cross sectional details of pipe bend after performing the experimental evaluation with internal pressure and in-plane bending loading. The evaluations were performed for inlet pigtail pipe bend design parameters of $R / r=5$ and $r / t=10$. The outer diameter (Fig. 14) and thickness variation (Fig. 16) are not uniform across the cross section of pipe bend. The impact on in-plane bending and internal pressure loading causes considerable deformation in internal diameter which is more in intrados region compared to extrados (Fig. 15).

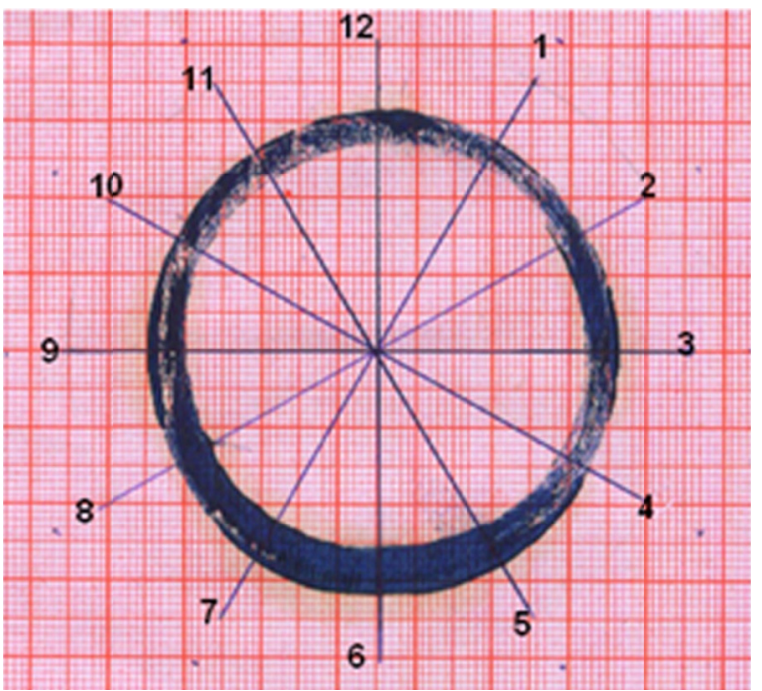

Fig. 13. Bend Profile after loading

\section{3a. Results comparison with FEA}

In the range of the pipe bend dimension ratio investigated, it was found that the failure life of the pipe bends with shape imperfection mainly depend on the bend factor. For lower bend factor pipe bend, the impact of shape imperfection was lesser than that of the pipe used with higher bend factor. The effect of geometry change becomes more significant when the pipes are thick. It may be observed that the FEA has produced results very

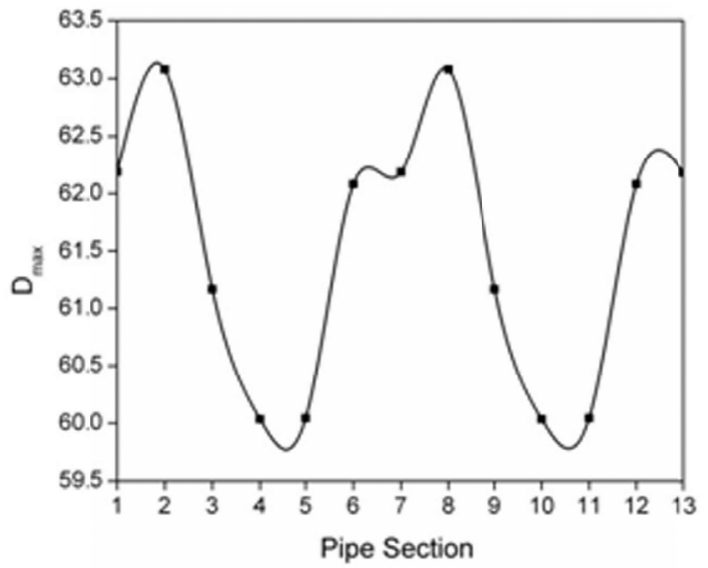

Fig. 14. $D_{\max }$ Variation

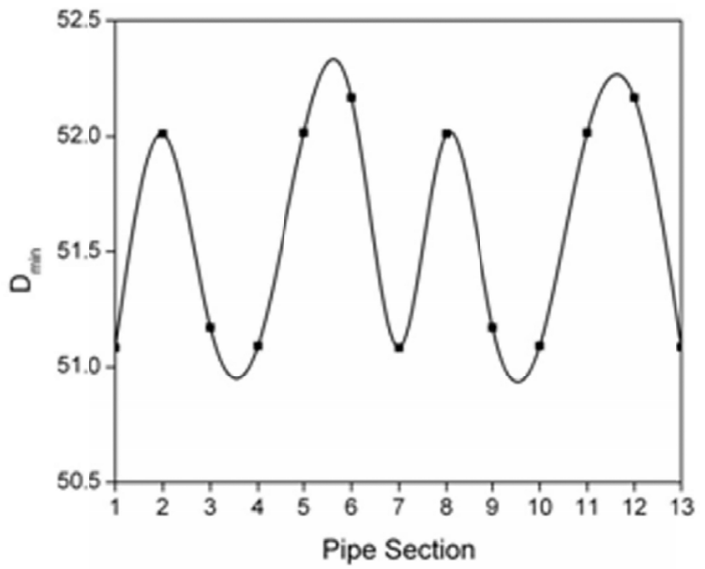

Fig. 15. $D_{\min }$ Variation

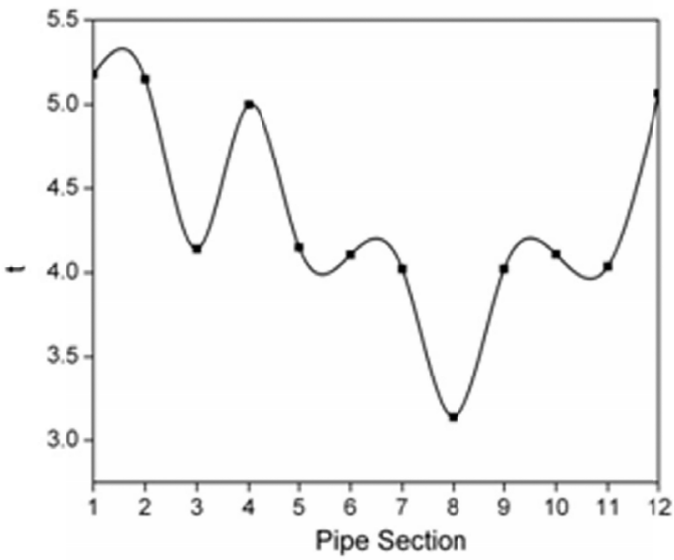

Fig. 16. Pipe thickness $(t)$ variation

close to those of experiment. The load-displacement curve for the specific design parameter of pipe bend geometry was plotted with experimental and FEA results (Fig. 17). In a nutshell, it can be said that the membrane stresses vary linearly with the thickness and the bending stresses vary with the square of thickness. The result of analysis showed that the FE analyses are lower bound when compared with the experimental solution. The limit loads for FEA and experimental are found to be $3.36 \%$ variation based on TES method. 


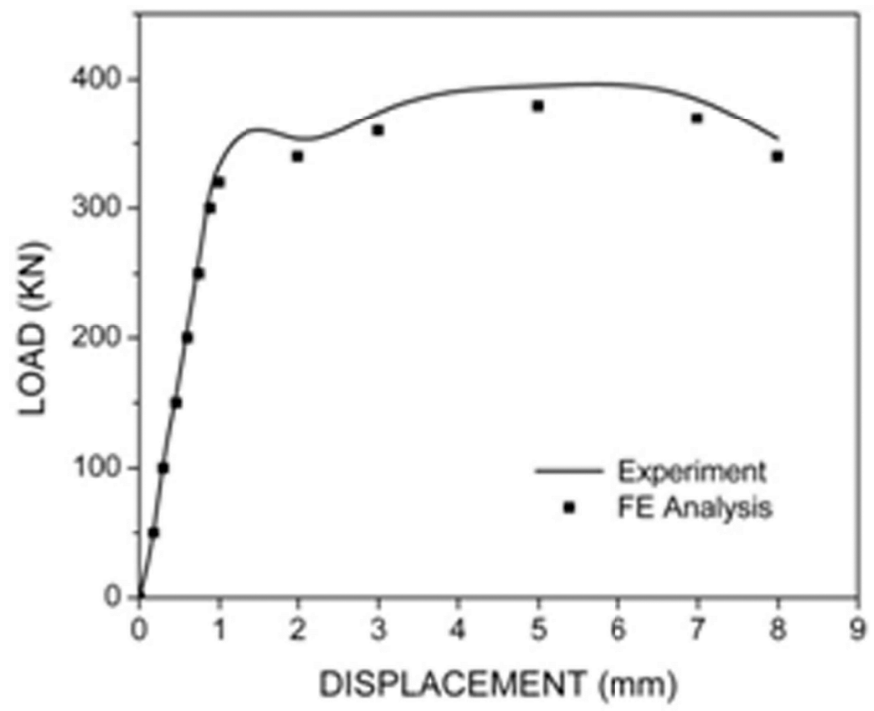

Fig. 17. Comparison of load deflection curve

\section{Conclusions}

The results of the FE study proved that for $90^{\circ}$ pipe bend, under in-plane bending moment produced not only an axial membrane stress component but also for axial and hoop bending stress components. The magnitudes of these stress components are the greatest at or near the crown in the cross-section and in the center of the pipe bend. From the analysis, it was identified that for in-plane bending, the limit load does not depend on the length of the attached straight pipe, when the ratio of the length to the mean radius of the pipe is greater than five. It was also identified that when the ratio is less than five, then the limit load decreases gradually with the decreasing length.

The pipe bend subjected to in-plane bending moment increases the cross-sectional moment of inertia due to ovalisation and therefore it stiffens at the intrados region of pipe bend and at the extrados the stiffness gets reduced, based on the moment load acting on the pipe bend. It was also identified that the application of internal pressure enhances the limit moment of a pipe bend slightly up to a certain limit, beyond which it starts falling with further increase in the internal pressure. It has been noted that the moment loading caused considerable cross section ovalisation in the thin walled pipe bend, when compared with internal pressure loading and the acceptability of pipe bend based on the operating conditions of the plant and loads acting on the pipe bend. The shape imperfection of ovality in pipe bends plays a major role for the acceptability of pipe bends. Limit load of pipe bend decreases with the increase in percent ovality $\left(C_{o}\right)$ due to decrease in bending stiffness at cross section. Further, the effect of internal pressure and limit moment depend on pipe bend factor $(h)$.

\section{Nomenclature}

$D$ : nominal pipe diameter,

$D_{\max }$ : maximum outside pipe diameter, $\mathrm{mm}$,

$D_{\min }:$ minimum outside pipe diameter, $\mathrm{mm}$,

$D_{o}$ : pipe outside diameter, $\mathrm{mm}$,

$t \quad$ : nominal thickness of pipe bend, mm,

$E \quad$ : elastic modulus, $\left(\mathrm{N} / \mathrm{mm}^{2}\right)$,

$t_{\max }:$ maximum pipe thickness, mm,

$t_{\min }$ : minimum pipe thickness, mm,

$\gamma \quad$ : Poisson's ratio,

$\sigma_{o} \quad$ : Limiting yield stress $\left(\mathrm{N} / \mathrm{mm}^{2}\right)$,

$P \quad$ : pipe internal pressure, $\left(\mathrm{N} / \mathrm{mm}^{2}\right)$,

$F \quad$ : applied force $(\mathrm{N})$,

$h \quad$ : bend factor.

\section{REFERENCES}

[1] H. Li, J. Wood, R. McCormack, R. Hamilton, Int. Jour. of Press. and Pip. 10, 154-160 (2013).

[2] T. Christo Michael, AR. Veerappan, S. Shanmugam, Eng. Frac. Mech. 105, 1-15 (2013).

[3] S. Sumesh, AR. Veerappan, S. Shanmugam, Proc. Wor. Con. Eng. Vol. II, London UK 2015.

[4] P. Ramaswami, P. Senthilvelmurugan, Int. Jour. of App. Eng. Res. 10, 38802-38807 (2015).

[5] TaeRyong Kim, Changkyun Oh, Int. Conf. on Nucl. Eng. I, Czech Republic 2014.

[6] Yoshio Urabe, Koji Takahashi, Hisanori Abe , J. Pres. Tech. 137, 041404 (2015).

[7] George E Varelis, Spyros A Karamanos, J. Pres. Tech. 137, 011401 (2014).

[8] Jin Weon Kim, Jour. of Pres. Tech. 131, 031210 (2009).

[9] Seok Pyo Hong, Joong Hyok An, Yun-Jae Kim, Int. Jour. of Mech. Scien 53, 526-535 (2011).

[10] Haofeng Chen, James Ure, Tianbai Li, Weihang Chen, Donald Mackenzie, Int.Jour. of Pres. and Piping 88, 213-222 (2011).

[11] Kim YJ, Oh CS, Int. Jour. of Pres. and Piping 83, 85-90 (2006).

[12] Kim YJ, Oh CS, Int. Jour. of Pres. and Piping 84, 177-14 (2007).

[13] Mohindar L. Nayyar, Piping Handbook, 2000, McGraw-Hill. 